\title{
Asymptotic stability of tri-trophic food chains sharing a common resource
}

\author{
Ivo Vrkoča ${ }^{\mathrm{a}}$, Vlastimil Křivan ${ }^{\mathrm{b}, \mathrm{c}}$ \\ ${ }^{a}$ Mathematical Institute, Czech Academy of Sciences, Žitná 25, 11567 Praha 1, Czech \\ Republic \\ ${ }^{b}$ Institute of Entomology, Biology Center, Czech Academy of Sciences, Branišovská 31, \\ 37005 Ceské Budějovice, Czech Republic \\ ${ }^{c}$ Department of Mathematics and Biomathematics, Faculty of Science, University of \\ South Bohemia, Branišovská 1760, 37005 České Budějovice, Czech Republic
}

\begin{abstract}
One of the key results of the food web theory states that the interior equilibrium of a tri-trophic food chain described by the Lotka-Volterra type dynamics is globally asymptotically stable whenever it exists. This article extends this result to food webs consisting of several food chains sharing a common resource. A Lyapunov function for such food webs is constructed and asymptotic stability of the interior equilibrium is proved. Numerical simulations show that as the number of food chains increases, the real part of the leading eigenvalue, while still negative, approaches zero. Thus the resilience of such food webs decreases with the number of food chains in the web.
\end{abstract}

Keywords: competition, food webs, Lyapunov function, resilience, stability

\section{Introduction}

Competition is one of the main driving forces reducing biodiversity in complex food webs. The "competitive exclusion principle" formulated by Gause [1] excludes coexistence of two species that compete for a single resource. Levin [2] proved that $n$ competing species cannot coexist at a population equilibrium if they are limited by less than $n$ limiting factors. As species are often limited by a few nutrients (e.g., phosphorus and/or nitrogen

\footnotetext{
Email addresses: vrkoc@math.cas.cz (Ivo Vrkoč), vlastimil.krivan@gmail.com (Vlastimil Křivan )
}

Preprint submitted to Mathematical Biosciences

September 29, 2015

(C) 2015. This manuscript version is made available under the Elsevier user license http://www.elsevier.com/open-access/userlicense/1.0/ 
in lakes) how is it then possible that many species do survive [3]? Several mechanisms explaining species coexistence were proposed. These include, but are not limited to non-equilibrium dynamics due to environmental [3] or internal [4] fluctuations in population dynamics, relative nonlinearity in species responses to competition $[5,6]$, predation on competing species $[7,8]$, or adaptive foraging $[9,10]$. These mechanisms fit into two broad categories [5]: (i) Stabilizing mechanisms that tend to increase negative intraspecific interactions relative to interspecific interactions (density dependent mechanisms, e.g., the logistic population growth,) and (ii) Equalizing mechanisms that tend to decrease average fitness differences between species. These latter mechanisms are often expressed through changes in evolutionary/behavioral traits.

The interplay of these two mechanisms on stability and persistence in di- and tri-trophic food webs were studied in [11]. That study was motivated by "the paradox of phytoplankton" where in lakes a large number of phytoplankton species survives on just one or two common resources (e.g., phosphorus) [3]. One of the food web modules considered assumed that each of the phytoplankton species was regulated by a specialized predator (e.g., a zooplankton species) (Figure 1). Thus the $n$ consumer species were competing for a single resource, but as each of them was consumed by a specialized predator, there were $n+1$ limiting factors so that the exclusion principle did not apply. Indeed, numerical simulations confirmed that all species could coexist. Such a food web is a generalization of a single tri-trophic food chain studied intensively in ecology $[12,13]$. Using a Lyapunov function, Harrison [14] proved that the interior equilibrium of the tri-trophic food chain is globally asymptotically stable whenever it exists. However, numerical simulations show that as the number of food chains sharing a common resource increases the stabilizing effect of the negative resource density dependence (modeled by the logistic resource growth) dilutes and numerical simulations are inconclusive with respect to the asymptotic stability of the interior equilibrium (more details are given in Discussion).

In this article we extend the result on the asymptotic equilibrium stability for a single tri-trophic food chain to many food chains sharing a common resource. Using a Lotka-Volterra type Lyapunov function we show that the resource density converges to an equilibrium and on the attractor each food chain dynamics are described by a Lotka-Volterra predator-prey model. However, these Lotka-Volterra predator-prey models are not independent as their trajectories satisfy a constraint. Using this information we prove in Sec- 
tion 2.1 that for two food chains sharing a common resource the population equilibrium is globally asymptotically stable. We extend this result in Section 2.2 where we prove that the population equilibrium is locally asymptotically stable for any number of food chains.

\section{Model}

We consider a tri-trophic food-web consisting of a common resource $(x)$, $n$ consumers $\left(y_{1}, \ldots, y_{n}\right)$, and top specialist predators $\left(z_{1}, \ldots, z_{n}\right)$ illustrated in Figure 1. Such a food web topology can describe e.g., a single plant species with several aphid species each of them parasitized by a specialized parasitoid [15]. The Lotka-Volterra conceptualization of population dynamics in such a food web is

$$
\begin{array}{rlr}
\frac{d x}{d t} & =r x\left(1-\frac{x}{K}\right)-\sum_{i=1}^{n} \lambda_{i} y_{i} x & \\
\frac{d y_{i}}{d t}=y_{i}\left(e_{i} \lambda_{i} x-m_{i}-\Lambda_{i} z_{i}\right) & i=1, \ldots, n \\
\frac{d z_{i}}{d t}=z_{i}\left(E_{i} \Lambda_{i} y_{i}-M_{i}\right) & i=1, \ldots, n,
\end{array}
$$

where $r$ is the resource specific growth rate, $K$ is the resource environmental capacity, $\lambda_{i}\left(\Lambda_{i}\right)$ is the consumer (predator) search rate for resource (consumer) $i, e_{i}\left(E_{i}\right)$ is the efficiency rate with which the resource (consumers) are converted to new consumers (predators), and $m_{i}\left(M_{i}\right)$ is the consumer (predator) mortality rate [11]. In what follows we assume that all these parameters are positive and we consider only solutions of (1) that are nonnegative (i.e., all initial conditions are positive). The interior equilibrium of (1) is

$$
\begin{array}{rlr}
x^{*}=K\left(1-\frac{1}{r} \sum_{i=1}^{n} \frac{\lambda_{i} M_{i}}{E_{i} \Lambda_{i}}\right) & \\
y_{i}^{*}=\frac{M_{i}}{E_{i} \Lambda_{i}} & i=1, \ldots, n \\
z_{i}^{*}=\frac{e_{i} \lambda_{i} x^{*}-m_{i}}{\Lambda_{i}} & i=1, \ldots, n .
\end{array}
$$


This equilibrium is positive provided the intrinsic per capita resource population growth rate is high enough so that

$$
r>\sum_{i=1}^{n} \frac{\lambda_{i} M_{i}}{E_{i} \Lambda_{i}}
$$

and the resource environmental carrying capacity satisfies

$$
K\left(1-\frac{1}{r} \sum_{i=1}^{n} \frac{\lambda_{i} M_{i}}{E_{i} \Lambda_{i}}\right)>\frac{m_{i}}{e_{i} \lambda_{i}}, \quad i=1, \ldots, n .
$$

In what follows we will assume the above two inequalities hold and we study stability of equilibrium (2).

First we observe that the resource density $x(t)$ converges to the equilib$\operatorname{rium} x^{*}$.

Proposition 1. Positive solutions of (1) are bounded and component $x(t)$ of every solution converges to the equilibrium $x^{*}$.

Proof. Let

$V=x-x^{*}-x^{*} \ln \frac{x}{x^{*}}+\sum_{i=1}^{n} \frac{1}{e_{i}}\left(y_{i}-y_{i}^{*}-y_{i}^{*} \ln \frac{y_{i}}{y_{i}^{*}}\right)+\sum_{i=1}^{n} \frac{1}{e_{i} E_{i}}\left(z_{i}-z_{i}^{*}-z_{i}^{*} \ln \frac{z_{i}}{z_{i}^{*}}\right)$.

Then $V\left(x^{*}, y_{1}^{*}, \ldots, y_{n}^{*}, z_{1}^{*}, \ldots, z_{n}^{*}\right)=0, V$ is non-negative and

$$
\frac{d V}{d t}=-\frac{r}{K}\left(x-x^{*}\right)^{2}
$$

along trajectories of model (1). Thus $V$ is a Lyapunov function and all trajectories of model (1) are bounded.

Let us consider a non-trivial solution of (1) and let us assume that $x(t)$ does not converge to $x^{*}$. There exists a sequence of times $t_{s} \rightarrow \infty$ and $\delta_{1}>0$ so that $\left|x\left(t_{s}\right)-x^{*}\right|>\delta_{1}$. Because the trajectory is bounded, its derivative is bounded as well and there exists $\delta_{2}>0$ such that $\left|x(t)-x^{*}\right|>\delta_{1} / 2$ for $t \in\left(t_{s}-\delta_{2}, t_{s}+\delta_{2}\right)$ and all $t_{s}$. Thus, (5) implies that $V$ cannot be non-negative for all $t$ 's, a contradiction with non-negativity of $V$.

Second, let us consider an $\omega$-limit solution $\left(x^{\omega}(t), y^{\omega}(t), z^{\omega}(t)\right)$ of $(1)$. It follows from Proposition 1 that $x^{\omega}(t)=x^{*}$ for every $t \in \mathbb{R}$ and therefore from (1)

$$
\sum_{i=1}^{n} \lambda_{i} y_{i}^{\omega}(t)=r\left(1-\frac{x^{*}}{K}\right)
$$


Moreover, the $\omega$-limit solution satisfies the following Lotka-Volterra system of paired equations

$$
\begin{array}{ll}
\frac{d y_{i}^{\omega}}{d t}=y_{i}^{\omega}\left(e_{i} \lambda_{i} x^{*}-m_{i}-\Lambda_{i} z_{i}^{\omega}\right) & i=1, \ldots, n \\
\frac{d z_{i}^{\omega}}{d t}=z_{i}^{\omega}\left(E_{i} \Lambda_{i} y_{i}^{\omega}-M_{i}\right) & i=1, \ldots, n .
\end{array}
$$

These equations are pairs of the Lotka-Volterra predator-prey equations so that on the attractor we have the following Lyapunov functions

$$
V_{i}=\frac{1}{e_{i}}\left(y_{i}^{\omega}-y_{i}^{*}-y_{i}^{*} \ln \frac{y_{i}^{\omega}}{y_{i}^{*}}\right)+\frac{1}{e_{i} E_{i}}\left(z_{i}^{\omega}-z_{i}^{*}-z_{i}^{*} \ln \frac{z_{i}^{\omega}}{z_{i}^{*}}\right), i=1, \ldots, n .
$$

We want to prove that the only $\omega$-limit solution of system (7) that satisfies

(6) is the constant solution coinciding with equilibrium (2). The case of a single food chain $(n=1)$ was studied in [14] so we begin with the case $n=2$.

\subsection{The case of two competing food chains.}

We start with a system consisting of two food chains with a common limiting resource $(x)$

$$
\begin{aligned}
\frac{d x}{d t} & =r x\left(1-\frac{x}{K}\right)-\lambda_{1} y_{1} x-\lambda_{2} y_{2} x \\
\frac{d y_{1}}{d t} & =y_{1}\left(e_{1} \lambda_{1} x-m_{1}-\Lambda_{1} z_{1}\right) \\
\frac{d y_{2}}{d t} & =y_{2}\left(e_{2} \lambda_{2} x-m_{2}-\Lambda_{2} z_{2}\right) \\
\frac{d z_{1}}{d t} & =z_{1}\left(E_{1} \Lambda_{1} y_{1}-M_{1}\right) \\
\frac{d z_{2}}{d t} & =z_{2}\left(E_{2} \Lambda_{2} y_{2}-M_{2}\right)
\end{aligned}
$$

All parameters in (9) are assumed to be positive and conditions (3) and (4) imply that the interior equilibrium (2) exists.

Theorem 1. The interior equilibrium (2) (with $n=2$ ) of (9) is globally asymptotically stable. 
Proof. We prove that the only $\omega$-limit solution $\left(x^{\omega}(t), y_{1}^{\omega}(t), y_{2}^{\omega}(t), z_{1}^{\omega}(t), z_{2}^{\omega}(t)\right)$ of trajectories starting from an interior point of $\mathbb{R}_{+}^{5}=\left\{\left(x_{1}, \ldots, x_{5}\right) \mid x_{1}>\right.$ $\left.0, \ldots, x_{5}>0\right\}$ is the interior equilibrium. The $\omega$-limit solution must satisfy $(6)$,

$$
\lambda_{1} y_{1}^{\omega}(t)+\lambda_{2} y_{2}^{\omega}(t)=c:=r\left(1-\frac{x^{*}}{K}\right) \quad \text { for all } t \in \mathbb{R} .
$$

Differentiating this equality and substituting, we get

$$
\lambda_{1} y_{1}^{\omega}(t)\left(e_{1} \lambda_{1} x^{*}-m_{1}-\Lambda_{1} z_{1}^{\omega}(t)\right)+\lambda_{2} y_{2}^{\omega}(t)\left(e_{2} \lambda_{2} x^{*}-m_{2}-\Lambda_{2} z_{2}^{\omega}(t)\right)=0 .
$$

Due to (7) we also know that the limit solution is periodic. Let $\left\{t_{n}\right\}_{n=1}^{\infty}$ be a sequence of times such that $z_{2}^{\omega}\left(t_{n}\right)=z_{2}^{*}$. Then (11) implies that $z_{1}^{\omega}\left(t_{n}\right)=z_{1}^{*}$. We remark that $y_{i}^{\omega}\left(t_{n}\right) \neq y_{i}^{*}, i=1,2$.

For $t$ 's close to but different from $t_{n}, z_{2}^{\omega}(t) \neq z_{2}^{*}$. In what follows we will show that $y_{1}^{\omega}\left(t_{n}\right)$ is independent of $t_{n}$, i.e., constant and equal to the equilibrium point. From (11) we get

$$
\frac{\lambda_{2} y_{2}^{\omega}(t)}{\lambda_{1} y_{1}^{\omega}(t)}=-\frac{e_{1} \lambda_{1} x^{*}-m_{1}-\Lambda_{1} z_{1}^{\omega}(t)}{e_{2} \lambda_{2} x^{*}-m_{2}-\Lambda_{2} z_{2}^{\omega}(t)}
$$

for $t$ 's close to but different from $t_{n}$ for all $n$. Using L'Hospital's rule we get

$$
\frac{\lambda_{2} y_{2}^{\omega}\left(t_{n}\right)}{\lambda_{1} y_{1}^{\omega}\left(t_{n}\right)}=-\lim _{t \rightarrow t_{n}} \frac{e_{1} \lambda_{1} x^{*}-m_{1}-\Lambda_{1} z_{1}^{\omega}(t)}{e_{2} \lambda_{2} x^{*}-m_{2}-\Lambda_{2} z_{2}^{\omega}(t)}=\frac{\Lambda_{1}\left(z_{1}^{\omega}\right)^{\prime}\left(t_{n}\right)}{\Lambda_{2}\left(z_{2}^{\omega}\right)^{\prime}\left(t_{n}\right)}
$$

Substituting derivatives of $z_{i}^{\omega}$ from (7) gives

$$
\frac{\lambda_{2} y_{2}^{\omega}\left(t_{n}\right)}{\lambda_{1} y_{1}^{\omega}\left(t_{n}\right)}=-\frac{\Lambda_{1} z_{1}^{\omega}\left(t_{n}\right)\left(E_{1} \Lambda_{1} y_{1}^{\omega}\left(t_{n}\right)-M_{1}\right)}{\Lambda_{2} z_{2}^{\omega}\left(t_{n}\right)\left(E_{2} \Lambda_{2} y_{2}^{\omega}\left(t_{n}\right)-M_{2}\right)}
$$

Substituting $y_{2}^{\omega}(t)=\left(c-\lambda_{1} y_{1}^{\omega}(t)\right) / \lambda_{2}, c=\frac{\lambda_{1} M_{1}}{E_{1} \Lambda_{1}}+\frac{\lambda_{2} M_{2}}{E_{2} \Lambda_{2}}$ and solving the resulting quadratic equation for unknown $y_{1}^{\omega}\left(t_{n}\right)$ we obtain two solutions: $y_{1}^{\omega}\left(t_{n}\right)=y_{1}^{*}$ and

$$
y_{1}^{\omega}\left(t_{n}\right)=\frac{\Lambda_{2} z_{2}^{*}\left(E_{1} M_{2} \Lambda_{1} \lambda_{2}+E_{2} M_{1} \Lambda_{2} \lambda_{1}\right)}{E_{1} \Lambda_{1}\left(E_{1} \Lambda_{1}^{2} \lambda_{2} z_{1}^{*}+E_{2} \lambda_{1} \Lambda_{2}^{2} z_{2}^{*}\right)} .
$$

Solution $y_{1}^{\omega}\left(t_{n}\right)=y_{1}^{*}$ corresponds to the equilibrium point $\left(y_{1}^{*}, z_{1}^{*}\right)$. Equality (10) implies that $y_{2}^{\omega}\left(t_{n}\right)=y_{2}^{*}$ for all $t_{n}$. Thus, the corresponding $\omega$-limit solution is the equilibrium. 
There is no $\omega$-limit solution corresponding to the other solution (12). Indeed, any non-constant periodic solution must attain two different values $y_{1}^{\omega}\left(t_{n}\right)$ and $y_{1}^{\omega}\left(t_{n+1}\right)$ (one which is larger and one which is smaller than $y_{1}^{*}$ ). However, we obtained only one such solution (12).

\subsection{The case of several competing food chains.}

In this section we prove local asymptotic stability of equilibrium (2) for any number of food chains. We use the following lemmas.

Lemma 1. There exists a constant $\mathcal{K}>0$ such that

$$
\max _{i=1, \ldots, n}\left\{y_{i}^{\omega}(t), z_{i}^{\omega}(t)\right\} \leq \mathcal{K}, \quad t \in \mathbb{R}
$$

for every $\omega$-limit solution $\left(y^{\omega}, z^{\omega}\right) \in \mathbb{R}^{2 n}$ of (1).

Proof. Every $\omega$-limit solution $\left(y^{\omega}(t), z^{\omega}(t)\right) \in \mathbb{R}^{2 n}$ of (1) is periodic and satisfies equality (6). As we consider only non-negative solutions, it follows from (6) that $y$-coordinates of the solution are bounded, i.e., $y_{i}^{\omega}(t)<\mathcal{K}_{1}$ for all $i=1, \ldots, n$ and all $t \in \mathbb{R}$. Now we prove that $z$-coordinates are bounded too.

For every food chain $(i=1, \ldots, n)$ there exists a sequence of times $t_{i, k} \rightarrow$ $\infty$ such that $z_{i}^{\omega}\left(t_{i, k}\right)=z_{i}^{*}$. Thus, from (8) it follows that for all $t_{i, k}$

$$
V_{i}\left(t_{i, k}\right)=\frac{1}{e_{i}}\left(y_{i}^{\omega}\left(t_{i, k}\right)-y_{i}^{*}-y_{i}^{*} \ln \frac{y_{i}^{\omega}\left(t_{i, k}\right)}{y_{i}^{*}}\right), \quad i=1, \ldots, n .
$$

As we already know that $y_{i}^{\omega}(t)$ are bounded and $V_{i} \geq 0$ is constant along $\left(y_{i}^{\omega}(t), z_{i}^{\omega}(t)\right)$ we get that $z_{i}^{\omega}(t), i=1, \ldots, n$ are bounded too. This concludes the proof.

The next result follows from the existence of the Lyapunov function $V_{i}$ defined by (8).

Lemma 2. There exists a function $\eta(\epsilon)$ defined for $\epsilon>0, \lim _{\epsilon \rightarrow 0} \eta(\epsilon)=0$, $\lim _{\epsilon \rightarrow \infty} \eta(\epsilon)=\infty$ such that if $\omega$-limit solution $\left(y_{i}^{\omega}(t), z_{i}^{\omega}(t)\right)$ satisfies

$$
\max _{i=1, \ldots, n} \sqrt{\left(y_{i}\left(t_{0}\right)-y_{i}^{*}\right)^{2}+\left(z_{i}\left(t_{0}\right)-z_{i}^{*}\right)^{2}}<\epsilon
$$

for some $t_{0} \in \mathbb{R}$ then

$$
\sup _{t \in \mathbb{R}} \max _{i=1, \ldots, n} \sqrt{\left(y_{i}^{\omega}(t)-y_{i}^{*}\right)^{2}+\left(z_{i}^{\omega}(t)-z_{i}^{*}\right)^{2}}<\eta(\epsilon) .
$$


The next lemma is a crucial part of the proof of local asymptotic stability of (2). We prove that in a small neighborhood of the equilibrium the only $\omega$-limit solution of model (1) is the equilibrium solution. The proof is by induction with respect to the number of food chains. In what follows we set

$$
a_{i}=e_{i} \lambda_{i} x^{*}-m_{i}, \quad b_{i}=\Lambda_{i}, \quad c_{i}=E_{i} \Lambda_{i}, \quad d_{i}=M_{i}, \quad \omega_{i}=\sqrt{a_{i} d_{i}} .
$$

We remark that $2 \pi / \omega_{i}$ is the approximate period of small oscillations in the Lotka-Volterra predator-prey model at the equilibrium [16].

Lemma 3. Let $\omega_{i} \neq \omega_{j}$ for $i \neq j, i, j=1, \ldots, n$. Then there exists $\varepsilon>0$ so that the only $\omega$-limit solution of model (1) satisfying

$$
\min _{i=1, \ldots, n, n} \inf _{t \in \mathbb{R}} \sqrt{\left(y_{i}^{\omega}(t)-y_{i}^{*}\right)^{2}+\left(z_{i}^{\omega}(t)-z_{i}^{*}\right)^{2}}<\varepsilon
$$

is the equilibrium solution $\left(x^{*}, y^{*}, z^{*}\right) \in \mathbb{R}^{2 n+1}$ given by (2).

Proof. The proof is by induction with respect to the number of food chains $n$.

For $n=1$ it follows from (6) that any $\omega$-limit solution $y_{1}(t)$ must be constant so the condition is met.

Let us assume that Lemma 3 holds for any system consisting of $n-1$ food chains and it does not hold for a system consisting of $n$ food chains. This means that there exists a sequence of $\omega$-limit solutions $\left\{y^{k}(t), z^{k}(t)\right\} \in \mathbb{R}^{2 n}$ of (7) and a non-empty set of indexes $\mathcal{I}=\left\{i_{1}, \ldots, i_{s}\right\}, 1 \leq s \leq n$ such that

$$
\inf _{t \in \mathbb{R}} \sqrt{\left(y_{j}^{k}(t)-y_{j}^{*}\right)^{2}+\left(z_{j}^{k}(t)-z_{j}^{*}\right)^{2}}<1 / k, \quad j \in \mathcal{I} .
$$

For all other indexes $\mathcal{J}=\{1, \ldots, n\} \backslash \mathcal{I}$ we have

$$
\min _{j \in \mathcal{J}} \inf _{t \in \mathbb{R}} \sqrt{\left(y_{j}^{k}(t)-y_{j}^{*}\right)^{2}+\left(z_{j}^{k}(t)-z_{j}^{*}\right)^{2}}>\delta
$$

for some $\delta>0$. Lemma 2 implies that for every $j \in \mathcal{J},\left(y_{j}^{k}(t), y_{j}^{k}(t)\right)$ are bounded and we can choose an uniformly converging subsequence whose limit is again a component of an $\omega$-solution different from the equilibrium solution.

There are two possibilities. First, not all components of the $\omega$-limit solution converge to the components of the equilibrium, i.e., $s<n$. In this case some components of the $\omega$-limit solution converge to the equilibrium 
point which contradicts the induction assumption that for $n-1$ food chains there are no $\omega$-limit solutions in the neighborhood of the equilibrium that are different from the equilibrium.

Second, all components $(s=n)$ of the $\omega$-limit solutions converge to the equilibrium point, i.e., for all $k \in \mathbb{N}$

$$
\min _{i=1, \ldots, n} \inf _{t \in \mathbb{R}} \sqrt{\left(y_{i}^{k}(t)-y_{i}^{*}\right)^{2}+\left(z_{i}^{k}(t)-z_{i}^{*}\right)^{2}}<1 / k .
$$

Using (14) we write system (7) in the form

$$
\begin{aligned}
y_{i}^{\prime} & =y_{i}\left(a_{i}-b_{i} z_{i}\right), \quad i=1, \ldots, n \\
z_{i}^{\prime} & =z_{i}\left(c_{i} y_{i}-d_{i}\right), \quad i=1, \ldots, n .
\end{aligned}
$$

Let $\varepsilon^{k}=\sqrt{\sum_{i=1}^{n}\left(y_{0 i}^{k}-y_{i}^{*}\right)^{2}+\left(z_{0 i}^{k}-z_{i}^{*}\right)^{2}}$ where $\left(y_{0 i}, z_{0 i}\right) \in \mathbb{R}^{2 n}$ are initial conditions for (16) and

$$
\begin{aligned}
& u_{i}^{k}(t)=\frac{y_{i}^{k}(t)-y_{i}^{*}}{\varepsilon^{k}}, \\
& v_{i}^{k}(t)=\frac{z_{i}^{k}(t)-z_{i}^{*}}{\varepsilon^{k}}
\end{aligned}
$$

where $u_{i}^{*}=y_{i}^{*}=d_{i} / c_{i}$ and $v_{i}^{*}=z_{i}^{*}=a_{i} / b_{i}$. The transformed variables satisfy

$$
\begin{aligned}
& \left(u_{i}^{k}\right)^{\prime}(t)=-b_{i} v_{i}^{k}(t)\left(\varepsilon^{k} u_{i}^{k}(t)+u_{i}^{*}\right), \quad i=1, \ldots, n \\
& \left(v_{i}^{k}\right)^{\prime}(t)=c_{i} u_{i}^{k}(t)\left(\varepsilon^{k} v_{i}^{k}(t)+v_{i}^{*}\right), \quad i=1, \ldots, n .
\end{aligned}
$$

As $k$ tends to infinity, $\varepsilon^{k}$ tends to 0 . Moreover since

$$
\begin{aligned}
& u_{0 i}^{k}=\frac{y_{i}^{k}(0)-y_{i}^{*}}{\varepsilon^{k}}, \\
& v_{0 i}^{k}=\frac{z_{i}^{k}(0)-z_{i}^{*}}{\varepsilon^{k}},
\end{aligned}
$$

$\sum_{i=1}^{n}\left(u_{0 i}^{k}\right)^{2}+\left(v_{0 i}^{k}\right)^{2}=1$, and we can choose a convergent subsequence of initial conditions that converges to $\left(\tilde{v}_{0 i}, \tilde{v}_{0 i}\right)$. The corresponding $\omega$-limit solutions converge to the solution of the linear system

$$
\begin{aligned}
& \left(u_{i}^{k}\right)^{\prime}(t)=-b_{i} v_{i}^{k}(t) u_{i}^{*}, \quad i=1, \ldots, n \\
& \left(v_{i}^{k}\right)^{\prime}(t)=c_{i} u_{i}^{k}(t) v_{i}^{*}, \quad i=1, \ldots, n .
\end{aligned}
$$


We define

$$
\tilde{u}_{i}(t)=\lim _{k \rightarrow \infty} u_{i}^{k}(t)=\tilde{u}_{0 i} \cos \left(t \omega_{i}\right)-\tilde{v}_{0 i} \frac{b_{i}}{c_{i}} \sqrt{\frac{d_{i}}{a_{i}}} \sin \left(t \omega_{i}\right)
$$

and

$$
\tilde{v}_{i}(t)=\lim _{k \rightarrow \infty} v_{i}^{k}(t)=\tilde{v}_{0 i} \cos \left(t \omega_{i}\right)+\tilde{u}_{0 i} \frac{c_{i}}{b_{i}} \sqrt{\frac{a_{i}}{d_{i}}} \sin \left(t \omega_{i}\right) .
$$

From (6) we get

$$
\sum_{i=1}^{n} \lambda_{i} u_{i}^{k}(t)=0
$$

and

$$
\sum_{i=1}^{n} \lambda_{i} \tilde{u}_{i}(t)=0
$$

The last equality can be written as

$$
\sum_{i=1}^{n}\left(\alpha_{i} \cos \left(\omega_{i} t\right)+\beta_{i} \sin \left(\omega_{i} t\right)\right)=0
$$

where $\alpha_{i}$ and $\beta_{i}$ depend on the initial conditions. By double differentiation we obtain

$$
-\sum_{i=1}^{n} \omega_{i}^{2}\left(\alpha_{i} \cos \left(\omega_{i} t\right)+\beta_{i} \sin \left(\omega_{i} t\right)\right)=0
$$

We add this equation with equation

$$
\omega_{1}^{2} \sum_{i=1}^{n}\left(\alpha_{i} \cos \left(\omega_{i} t\right)+\beta_{i} \sin \left(\omega_{i} t\right)\right)=0
$$

to get

$$
\sum_{i=2}^{n}\left(\omega_{1}^{2}-\omega_{i}^{2}\right)\left(\alpha_{i} \cos \left(\omega_{i} t\right)+\beta_{i} \sin \left(\omega_{i} t\right)\right)=0 .
$$

Again, by double differentiation we obtain

$$
-\sum_{i=2}^{n} \omega_{i}^{2}\left(\omega_{1}^{2}-\omega_{i}^{2}\right)\left(\alpha_{i} \cos \left(\omega_{i} t\right)+\beta_{i} \sin \left(\omega_{i} t\right)\right)=0 .
$$


We add this equation with equation

$$
\omega_{2}^{2} \sum_{i=2}^{n}\left(\alpha_{i} \cos \left(\omega_{i} t\right)+\beta_{i} \sin \left(\omega_{i} t\right)\right)=0
$$

to get

$$
\sum_{i=3}^{n}\left(\omega_{1}^{2}-\omega_{i}^{2}\right)\left(\omega_{2}^{2}-\omega_{i}^{2}\right)\left(\alpha_{i} \cos \left(\omega_{i} t\right)+\beta_{i} \sin \left(\omega_{i} t\right)\right)=0 .
$$

Repeating double derivation and summation $n$ times we finally get

$$
\prod_{i=1}^{n-1}\left(\omega_{i}^{2}-\omega_{n}^{2}\right)\left(\alpha_{n} \cos \left(\omega_{n} t\right)+\beta_{n} \sin \left(\omega_{n} t\right)\right)=0 .
$$

As the last term cannot be equal to $0\left(\omega_{n}=\sqrt{a_{n} d_{n}}>0\right.$ and considered solutions are non-trivial), we obtain a contradiction with the assumption that $\omega_{i} \neq \omega_{j}$ for $i \neq j$. It follows that the lemma holds for $n$ too.

Theorem 2. Let $\omega_{i} \neq \omega_{j}$ for $i \neq j, i, j=1, \ldots, n$. Then the interior equilibrium $\left(x^{*}, y^{*}, z^{*}\right) \in \mathbb{R}_{+}^{2 n+1}$ of model (1) is locally asymptotically stable.

Proof. Because $\left(x^{*}, y^{*}, z^{*}\right)$ is a stable equilibrium of $(1)$, solutions that start close to this point stay in a vicinity of this point. If there was a solution of (1) such that for some $i \in\{1, \ldots, n\}, y_{i}(t), z_{i}(t)$ did not converge to the equilibrium point, there would exist an $\omega$-solution in a neighborhood of the equilibrium different from the equilibrium. This contradicts Lemma 3.

\section{Discussion}

In this article we proved that species in a food web consisting of several tri-trophic food chains with a shared resource can coexist in a locally asymptotically stable interior equilibrium. In particular, when there are only two such food chains, the interior equilibrium is globally asymptotically stable. These results extend the work of Harrison [14] who proved that the interior equilibrium of a single tri-trophic food chain is globally asymptotically stable whenever it exists.

The food web with $n$-food chains that share a common resource was considered in [11]. It was suggested there that population dynamics converge to the interior equilibrium whenever this equilibrium exists. The problem 
is that numerical simulations document such convergence clearly for low dimensional systems (i.e., one, two, or three food chains) but for many food chains convergence is not that clear. For example, numerical simulations in Mathematica 10 as those in Figure 2 show small persistent fluctuations of trajectories around the equilibrium for a food web consisting of 50 food chains. From the ecological point of view the reason is that stability of the interior equilibrium is driven by negatively density dependent resource growth (i.e., the per capita population growth rate decreases as the population density increases) modeled by the logistic equation. As the number of food chains increases, this negative density dependence "dilutes" which is manifested by increasing values of the real part of the leading eigenvalue associated with consumer and predator species (Figure 3). Albeit these largest real parts are still negative, they are very small in absolute value for large number of food chains. Thus, the bottom up regulation of the food web gets weaker with the number of food chains and the consumer and resources are more prone to oscillations after a disturbation. This shows that ecological resilience [17], defined as the rate with which a system returns to its equilibrium after a perturbation, decreases with the number of food chains in the food web. The resilience is measured as the absolute value of the real part of the dominant eigenvalue and the inverse of resilience is often termed the return time. Our results show that the return time increases with the number of food chains supported by a single resource. This shows that although mathematically stable, the real food webs consisting of several food chains sharing a common resource will be oscillating around the equilibrium.

We proved that the global asymptotic convergence to equilibrium holds for any parameter values for which the interior population equilibrium for two food chains exists. For the case of more than 2 food chains we were unable to extend this proof and we used a slightly different approach based on linearization of the original system at the interior equilibrium. This linearization led to the requirement that the parameters of the model are such that the periods of the oscillations of the linearized model are pairwise different (i.e., $\omega_{i} \neq \omega_{j}$ in Theorem 2). It is likely that the result holds even without this additional assumption and the stability is global.

\section{Acknowledgement}

We thank two anonymous reviewers for their thoughtful suggestions. Support provided by the Institute of Entomology (RVO:60077344) is acknowl- 
edged. This work was partly conducted while VK was a Sabbatical Fellow at the Mathematical Biosciences Institute, an Institute sponsored by the National Science Foundation under grant DMS 0931642.

\section{References}

[1] G. F. Gause, The struggle for existence, Williams and Wilkins, Baltimore, 1934.

[2] S. A. Levin, Community equilibria and stability: An extension of the competitive exclusion principle, American Naturalist 104 (1970) 413-23.

[3] G. E. Hutchinson, The paradox of the plankton, American Naturalist 95 (1961) 137-45.

[4] R. A. Armstrong, R. McGehee, Competitive exclusion, American Naturalist 115 (1980) 151-70.

[5] P. Chesson, Mechanisms of maintenance of species diversity, Annual Review of Ecology and Systematics 31 (2000) 343-66.

[6] P. Chesson, General theory of competitive coexistence in spatiallyvarying environments, Theoretical Population Biology 58 (2000) 211-37.

[7] L. Slobodkin, Experimental populations of hydrida, Journal of Animal Ecology 33 (1964) 131-48.

[8] R. T. Paine, A note on trophic complexity and community stability, American Naturalist 103 (1969) 91-3.

[9] V. Křivan, Competitive coexistence caused by adaptive predators, Evolutionary Ecology Research 5 (2003) 1163-82.

[10] V. Křivan, Behavioral refuges and predator-prey coexistence, Journal of Theoretical Biology 339 (2013) 112-21.

[11] V. Křivan, Competition in di- and tri-trophic food web modules, Journal of Theoretical Biology 343 (2014) 127-37.

[12] N. G. Hairston, F. E. Smith, L. B. Slobodkin, Community structure, population control, and competition, American Naturalist 94 (1960) 421-5. 
[13] L. Oksanen, S. D. Fretwell, J. Arruda, P. Niemelä, Exploitation ecosystems in gradients of primary productivity, American Naturalist 118 (1981) 240-61.

[14] G. H. Harrison, Global stability of food chains, American Naturalist 114 (1979) 455-7.

[15] C. J. Melian, V. Krivan, F. Altermatt, P. Stary, L. Pellissier, F. De Laender, Heterogeneous and multi-trophic metacommunities shape the biogeography of ecological networks, American Naturalist 85 (2015) 157-68.

[16] A. J. Lotka, Elements of mathematical biology, Dover Publications, Inc., New York, USA, 1956.

[17] R. M. May, Stability and complexity in model ecosystems, Princeton University Press, Princeton, NJ, USA, 1973. 


\section{Figures}

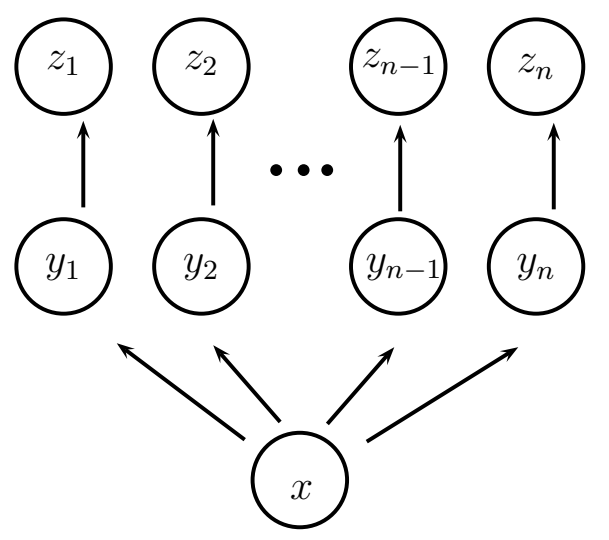

Figure 1: Food web with $n$ tri-trophic chains sharing a common resource. 
Figure 2: This plot shows simulations of model (1) with 50 consumer and predator species $(n=50$ in model (1)). For clarity only three trajectories for consumers and predators are shown. Simulations were done in Mathematica 10 using NDSolve function. Parameters of model (1) were generated at random from the normal distribution $N(\nu, \sigma)$ with mean $\nu$ and standard deviation $\sigma: r=N(2,0.4), \lambda=N(0.1,0.02), \Lambda=N(1,0.2), m=N(0.2,0.04)$, $M=N(0.1,0.02), e=N(0.5,0.1), E=N(0.5,0.1), K=N(50,10)$. 


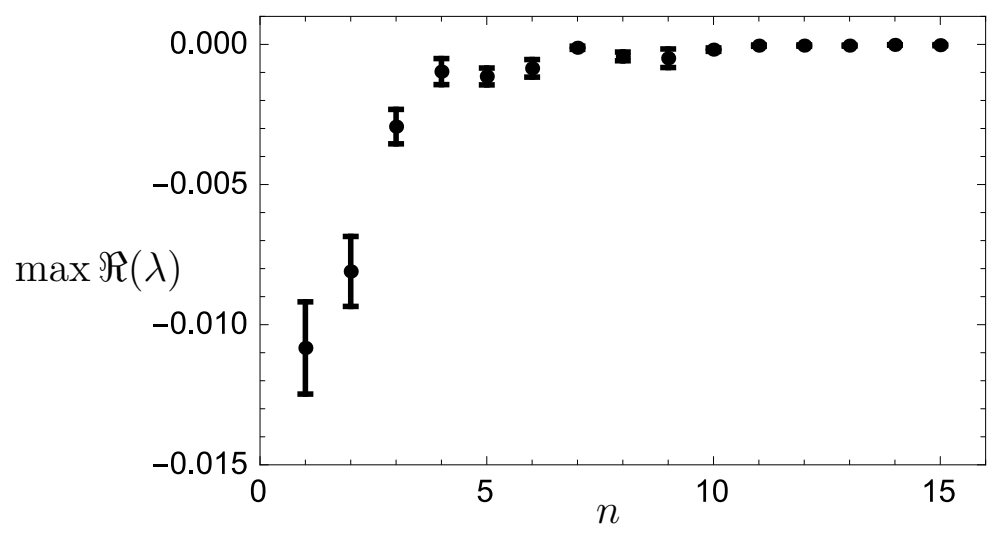

Figure 3: Dependence of the largest real part eigenvalue of model (1) on the number of food chains $n$ in the food web. Dots show averages of 30 simulations and bars are \pm 1 standard error. Parameters of model (1) were generated at random from the normal distribution $N(\nu, \sigma)$ with mean $\nu$ and standard deviation $\sigma$ same as in those used in Figure 2. 